\title{
Metallographische Mitteilungen aus dem Institut für anorganische Chemie der Universität Göttingen.
}

XVII.

\section{Über die Verbindungen des Eisens mit Silicium. \\ Von}

\author{
W. Guertler und G. Tammann.
}

Mit 2 Figuren im Text und 1 Tafel.

Siliciumeisenlegierungen sind wiederholt Gegenstand der Untersuchungen gewesen, und von verschiedenen Autoren ist die Existenz folgender Eisensiliciumverbindungen, deren Zusammensetzung den Formeln $\mathrm{Fe}_{2} \mathrm{Si}, \mathrm{Fe}_{3} \mathrm{Si}_{2}, \mathrm{FeSi}$ und $\mathrm{FeSi}_{2}$ entsprechen soll, behauptet worden.

Beim Behandeln von siliciumhaltigem Eisen mit verschiedenen Lösungsmitteln hinterbleibt ein Rückstand, welcher wiederholt näher untersucht wurde.

$\mathrm{H}_{\mathrm{AHN}}{ }^{1}$ schmolz Eisen mit $12 \%$ Silicium, $60 \%$ Natrium, ferner Flufsspat, Salmiak und Kochsalz zusammen, behandelte die Schmelze mit verdünnter Salzsäure und erhielt so einen Rückstand von der Zusammensetzung $\mathrm{Fe}_{2} \mathrm{Si}$. Denselben Körper erhielt $\mathrm{GIN}^{2}$ durch Zusammenschmelzen von Eisen mit Alkalisilikaten und Auslaugen der erkalteten Schmelze, OsmoND ${ }^{3}$ durch Einwirkung von Sublimatlösung auf ein $12 \%$ Si enthaltendes Eisen, ferner Moissan ${ }^{4}$ und Lebead ${ }^{5}$, welche bis $20 \%$ iges Siliciumeisen mit verdünnter Salpetersäure behandelten, und endlich Carnot und GouTaL ${ }^{6}$ als Rückstand bei der Einwirkung von kalter $5 \%$ iger Schwefelsäure auf siliciumhaltiges

1 Liebigs Ann. 129 (1864), 57.

2 Patentblatt. Klasse 18, Nr. 143506.

${ }^{3}$ Compt. rend. 113 (1891), 474.

4 Compt. rend. 121 (1895), 621. - Ann. chim. phys. [7] 9 (1896), 289.

5 Compt. rend. 131 (1900), 583.

${ }^{6}$ Compt. rend. 126 (1898), 1240. - Ann. des Mines [9] 18, 268. 
Eisen. Aufserdem hat Lebeau 1. c. durch Zusammenschmelzen von $300 \mathrm{~g}$ Kupfersilicium mit $150 \mathrm{~g}$ Eisen und nachheriges Behandeln der Schmelze mit verdünnter Salpetersäure einen Rückstand erhalten, dessen Zusammensetzung ebenfalls der Formel $\mathrm{Fe}_{2} \mathrm{Si}$ entsprach.

Es sind danach aus Siliciumeisen von verschiedenem Siliciumgehalt, bis zu $20 \%$, durch Behandeln mit verschiedenen Lösungsmitteln Rückstände von ein und derselben Zusammensetzung $\mathrm{Fe}_{2} \mathrm{Si}$ erhalten worden.

Über eine zweite Verbindung $\mathrm{Fe}_{2} \mathrm{Si}$ liegen folgende Angaben vor: FREMY ${ }^{1}$ erhielt dieselbe durch Einwirkung von Chlorsiliciumdampf auf erhitztes Eisen in kleinen regulären Oktaedern, unlöslich in Königswasser.

LEBEAU ${ }^{2}$ erhielt diese Verbindung als Rückstand bei der Einwirkung von $20 \%$ iger Salpetersäure auf eine Schmelze von Eisen mit viel überschüssigem Kupfersilicium.

$\mathrm{H}_{\mathrm{AHN}}$ (1.c.) erhielt beim Behandeln von $\mathrm{Fe}_{2} \mathrm{Si}$ mit verdünnter Flufssäure einen Rückstand von der Zusammensetzung FeSi. Die Homogenität dieses Rückstandes ist nicht bewiesen worden. Dasselbe gilt von einem Rückstande, den CarNot und Goutal (l.c.) durch Eintragen von Eisensilicium mit $10-14 \% \mathrm{Si}$ in $7 \%$ ige Salzsäure, Kochen bis zum Aufhören der Gasentwicklung und Entfernung der magnetischen Teile des Rückstandes durch einen Magneten erhielten. Auch dieser Rückstand besafs die Zusammensetzung FeSi.

LEBEAU $^{3}$ gibt an, dafs durch Schmelzen von FeSi mit Silber beim Abkühlen der Schmelze $\mathrm{Si}$ und die Verbindung $\mathrm{Fe}_{2} \mathrm{Si}$ aus der silberreichen Schmelze kristallisieren.

Die Angaben von Fremy und Lebeat sprechen wohl mit ziemlicher Entschiedenheit für die Existenz der Verbindung FeSi.

Aufser diesen beiden Verbindungen $\mathrm{Fe}_{2} \mathrm{Si}$ und $\mathrm{FeSi}$ ist noch die Existenz der Verbindungen $\mathrm{Fe}_{3} \mathrm{Si}_{2}$ und $\mathrm{FeSi}_{2}$ behauptet worden.

De Chatmot erbielt aus 25 bis $50 \%$ Si- haltigem Eisen Rückstände, deren Zusammensetzung den Formeln $\mathrm{Fe}_{3} \mathrm{Si}_{2}$ und $\mathrm{FeSi}_{2}$ entsprach, und LEBEAU ${ }^{3}$ glaubte die Verbindung $\mathrm{FeSi}_{2}$ erhalten zu

1 Wurmz, Dictionnaire de Chimie I. 2, S. 1417. 1418.

2 Compt. rend. 128 (1899), 933.

3 Compt. rend. 133 (1901), 1008.

4 Am. Chem. Journ. 18, 118. 
haben. Gründe für die Homogenität dieser Rückstände sind nicht angegeben worden.

Da somit unsere Kenntnis der Eisensiliciumverbindungen sich durchweg auf eine Reihe von Rückstandsanalysen gründet, war es wünschenswert, dieselbe durch Ausarbeitung des Zustandsdiagrammes der Eisensiliciumlegierungen sicherer zu fundieren.

\section{Das Versuchsverfahren.}

Die Eisensiliciumlegierungen wurden durch Zusammenschmelzen von Flufs-Eisen, welchen wir nebst den Angaben über seine $\mathrm{Zu}$ sammensetzung Herrn Direktor Dr. E. Ehrensberger von der Firma Krupp verdanken, mit Silicium, welches von dem Konsortium für elektrochemische Industrie in Nürnberg bezogen wurde, hergestellt.

Das Eisen enthielt:

$\begin{array}{lc}\mathrm{C} & 0.07 \% \\ \mathrm{Si} & 0.06 \% \\ \mathrm{Mn} & 0.10 \% \\ \mathrm{P} & <0.01 \% \\ \mathrm{~S} & 0.019 " \\ \mathrm{Cu} & 0.015,\end{array}$

Die Analyse des käuflichen Siliciums wurde in folgender Weise ausgeführt: Das feingepulverte Silicium wurde in geschmolzenes Natronhydrat, das sich in einem Silbertiegel befand, vorsichtig eingetragen. Nach fünfstündiger Einwirkung wurde die Schmelze mit Wasser behandelt und von einem Rückstande, der $2.86 \%$ betrug, abfiltriert. Die Menge desselben verringerte sich durch Abrauchen mit Flufssäure auf $1.27 \%$. Diese Gewichtsabnahme wurde als $\mathrm{SiO}_{2}$ in Abrechnung gebracht. Im Filtrat wurden nach den bekannten Methoden Silicium, Eisen und Aluminium bestimmt. Es ergab sich so folgende Zusammenstellung des angewandten Siliciums:

$$
\begin{aligned}
& \mathrm{Si} \quad 98.07 \% \\
& \mathrm{Fe} \quad 0.95 \text {, } \\
& \text { Al } 0.39, \\
& \text { Rückstand } 1.27 \text {, }
\end{aligned}
$$

Zur Herstellung der Legierungen wurden abgewogene unregelmärsig geformte Stücke des Siliciums und Eisenzylinder in ein Probierrohr aus Porzellan von $14 \mathrm{~mm}$ innerem Durchmesser, $10 \mathrm{~cm}$ 
Länge und $1.5 \mathrm{~mm}$ Wandstärke gebracht, so dals keine Verklemmung der im Rohr geschichteten Stücke eintreten konnte, dann durch Erhitzen des Probierrohres im elektrisch geheizten Kohlerohr zusammengeschmolzen und mit dem Schutzrohr des Thermoelementes sorgsam verrührt.

Das flüssige Silicium und die siliciumreichsten Schmelzen durchfressen in wenigen Minuten das Porzellanschutzrohr des Thermoelementes, worauf die leichttlüssige Schmelze zu den Drähten dringt. Infolgedessen mufste zur Aufnahme der Abkühlungskurven der Schmelzen mit über $75 \% \mathrm{Si}$ das Porzellanrohr des Thermoelementes durch eine Schicht Platinblech und Magnesiumoxyd wie bei der Untersuchung der Manganeisenschmelzen (siehe die vorstehende Arbeit von Levin und Tammans) geschützt werden.

Obwohl die Schmelzungen in einer Stickstoffatmosphäre vorgenommen wurden, so findet doch, besonders bei den siliciumreicheren Schmelzen eine nicht unerhebliche Änderung der Konzentration dadurch statt, dals einerseits das Silicium sich in der Stickstoffatmosphäre oberflächlich mit einem weifsen Anflug ${ }^{1}$ überzieht, andererseits die Schmelzen, je siliciumreicher sie sind, desto stärker auf das Porzellan wirken, indem sich offenbar ein Teil des Siliciums auf Kosten des Porzellans oxydiert und mit diesem eine mit dem Metall sich nicht mischende Schlacke liefert. Es war deutlich zu beobachten, dafs durch Einwirkung der siliciumreichen Schmelzen die Wände des Porzellanrohres, die mit der Schlacke in Berührung kamen, dünner geworden und mit einer grauen Schlacke bedeckt waren.

Um die durch diese Nebenreaktionen bedingten Gewichtsverluste der einzelnen Schmelzen zu bestimmen, wurden nach beendetem Versuch die erhaltenen Reguli, von denen die anhaftende Schlacke und der weilse Anflug leicht entfernt werden konnten, abermals gewogen. In nachfolgender Tab. 1 finden sich die Gewichtsprozente $\mathrm{Si}$, wie sie weiter unten nach Einführung der Korrekturen festgelegt wurden, die eingewogenen Mengen und unter der Rubrik "Abbrand" der gefundene Gewichtsverlust während des Versuches.

Um festzustellen, ob die Gewichtsabnahme ganz auf Rechnung des Verlustes an Silicium zu setzen sei, wurden die Reguli mit

1 Nach Deville und WöHLer, Ann. d. Pharm. 110, 248, und nach Sohürzenberoer und Colson, Compt. rend. 92, 1508, bedeckt sich glïhendes Silicium im Stickstoff oberflächlich mit einem weifsen flockigen Siliciumnitrid. 
Tabelle 1.

\begin{tabular}{c|c|c|c}
$\begin{array}{c}\text { Gewichtsprozent } \\
\mathrm{Si}\end{array}$ & $\begin{array}{c}\text { Eingewogene } \\
\text { Menge in } \mathrm{g}\end{array}$ & $\begin{array}{c}\text { Abbrand } \\
\text { in } \mathrm{g}\end{array}$ & $\begin{array}{c}\text { Volumina der } \\
\text { Schmelzen in ccm }\end{array}$ \\
\hline 0.06 & 19.93 & 0.1 & 2.57 \\
5.58 & 18.17 & 0.05 & 2.73 \\
11.3 & 17.95 & 0.02 & 2.87 \\
14.6 & 17.11 & 0.08 & 2.96 \\
18.8 & 16.75 & 0.0 & 3.03 \\
24.4 & 16.15 & & \\
27.3 & 16.10 & 0.1 & 3.13 \\
31.6 & 15.64 & 0.2 & 319 \\
41.5 & 14.38 & 0.3 & 3.27 \\
52.8 & 13.32 & 0.25 & 3.41 \\
64.6 & 12.09 & 0.4 & 3.57 \\
73.2 & 11.67 & 0.55 & 3.70 \\
79.8 & 11.19 & 0.6 & 3.79 \\
98.1 & 10.03 & 0.5 & 3.85 \\
& & 0.7 & 3.97
\end{tabular}

17.5, 33.0 und 66.2 eingewogenen Gewichtsprozenten Silicium analysiert. Hierzu wurden die Legierungen fein gepulvert und 5 Stunden im Silbertiegel mit Natron geschmolzen. Darauf wurden das Silicium und das Eisen wie oben bestimmt.

In Tab. 2 sind in der Rubrik, "gefunden" die Resultate der Analysen verzeichnet. In der Rubrik ,abgewogen" ist die $\mathrm{Zu}$ sammensetzung der Gemenge vor der Schmelzung angegeben. In der Rubrik „berechnet" finden sich die Konzentrationen, welche sich bei Berücksichtigung der weiter oben gegebenen Zusammensetzung des Ausgangsmaterials und des Abbrandes unter der Annahme, dafs nur Silicium in die Schlacke geht, ergeben. Man ersieht, dafs merkliche Abweichungen $\mathrm{zwischen}$ den so berechneten und den richtigen Konzentrationen erst bei höherem Siliciumgehalt auftreten.

Tabelle 2.

Gewichtsprozente Silicium.

\begin{tabular}{c|c|c}
\hline Abgewogen & Berechnet & Gefunden \\
\hline 17.5 & 17.4 & 17.43 \\
33.0 & 31.6 & 31.51 \\
66.2 & 64.6 & 64.00
\end{tabular}


Bei den Schmelzversuchen wurde stets die Abkühlungs- und Erhitzungskurve doppelt aufgenommen, indem nach der ersten Abkühlung sogleich wieder erhitzt, dann abermals abgekühlt und erhitzt wurde. In Übereinstimmung mit dem eben Gesagten fielen trotz der geringen Konzentrationsverschiebungen während der Versuche die beiden Abkühlungs- und die beiden Erhitzungskurven innerhalb der Versuchsfehler zusammen. Nur wenn infolge ungenügenden Rührens die Homogenität der Schmelze nicht erreicht war, wichen die Kurven voneinander $a b$, und die Bestimmungen wurden dann nach erneuter Durchmischung der Schmelze fortgesetzt, bis die letzten vier von den bestimmten Kurven in befriedigender Weise miteinander übereinstimmten. Nachdem dies erreicht war, war auch die Struktur der erkalteten Reguli an allen Stellen dieselbe.

Aus je zwei Abkühlungs- und zwei Erhitzungskurven wurden die Temperaturen des Beginns und des Endes der Kristallisation resp. Schmelzung entnommen und in den folgenden Tabellen 3, 4 und 5 zusammengestellt. Die Temperaturen sind sämtlich mittels des von Holborn, WiEN und $\mathrm{DAY}^{2}$ bestimmten Schmelzpunktes von Nickel $=1484^{\circ}$ und Gold $=1064^{\circ}$ auf die Skala des Luftthermometers bezogen.

Bei den Schmelzen des reinen Eisens und der Legierungen mit einem Siliciumgehalt bis zu 34.7 Atomproz. Silicium zeigen die Abkühlungs- und Erhitzungskurven nur eine einmalige Verzögerung der Abkühlungs- resp. Erhitzungsgeschwindigkeit. Die Daten für diese Legierungen finden sich in Tabelle 3 zusammengestellt.

(S. Tabelle 3, S. 169.)

Mit Hilfe der Abkühlungsgeschwindigkeit $a$ und der Erhitzungsgeschwindigkeit $e$, ausgedrückt in Graden pro Sekunde, sind aus den Temperaturen $t_{a}$ und $t_{e}$ die wahren Temperaturen der Schmelzen $t_{o}$ zu Beginn der Kristallisation und ebenso die wahren Temperaturen $t^{\prime}{ }_{o}$ bei Beendigung der Kristallisation aus $t^{\prime}{ }_{a}$ und $t^{\prime}{ }_{e}$ nach der Formel:

$$
t_{o}=t_{a}+\frac{a}{a+e}\left(t_{e}-t_{a}\right)
$$

berechnet, wie in $\mathrm{Bd}$. 45, 207 dieser Zeitschrift ausgeführt wurde. Die Temperaturintervalle $\Delta t$, innerhalb deren die Kristallisation der

1 Wied. Ann. 56 (1895), 360. - Drudes Ann. 2 (1900), 535. 


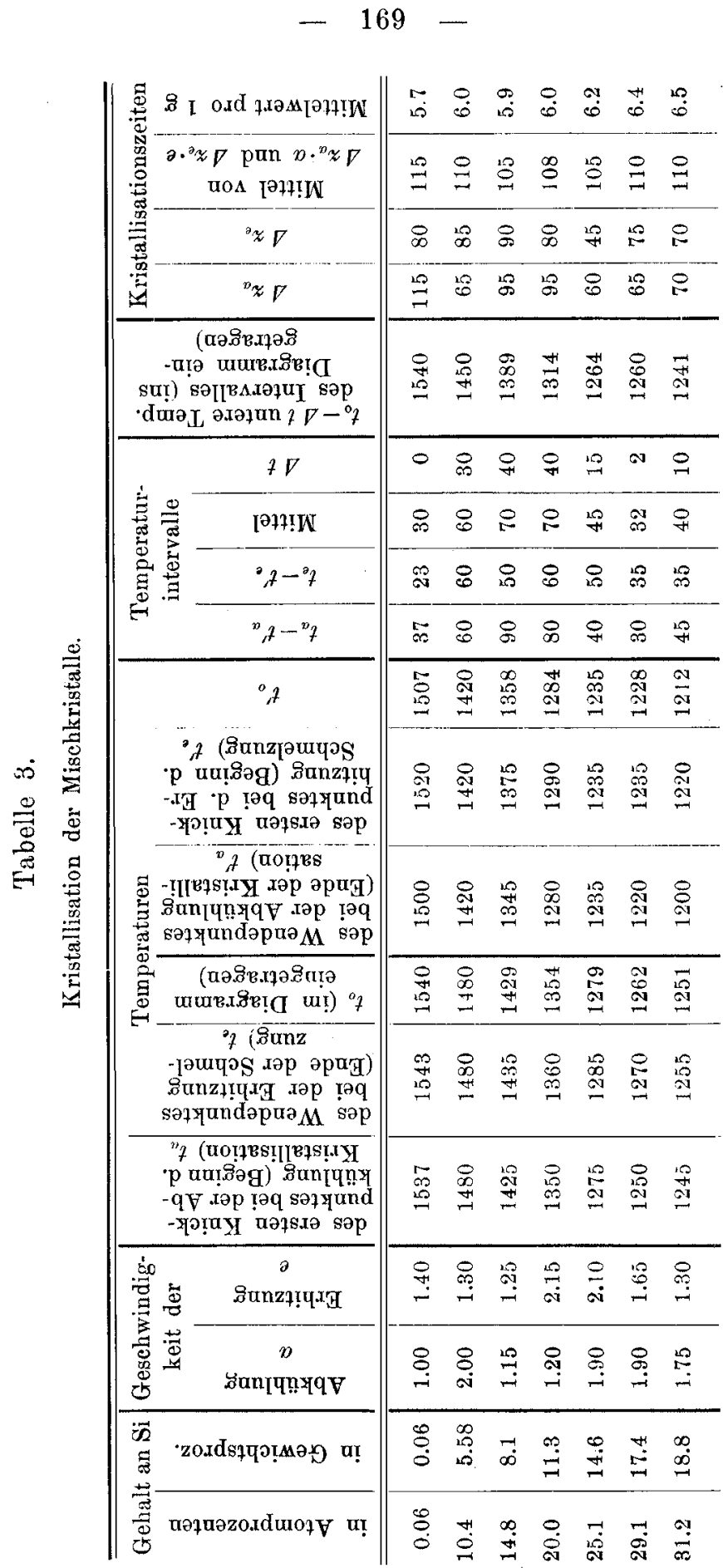


Mischkristalle sich vollzog, sind aus den Temperaturen $t_{o}$ und $t^{\prime}{ }_{o}$ (Tabelle 3) in derselben Weise wie in der vorstehenden Arbeit von Levin und TAMMaNN (S. 170) abgeleitet worden.

Aufserdem findet sich noch die Zeitdauer der Kristallisation bei der Abkühlung $\left(\Delta z_{a}\right)$ und bei der Erhitzung $\left(\Delta z_{e}\right)$ in Sekunden angegeben. Reduziert man diese auf gleiche Abkühlungs- und Erhitzungsgeschwindigkeiten, so erhält man die Werte $\Delta z_{a} \cdot a$ und $A z_{e} \cdot e$, deren Mittel in der Tabelle verzeichnet sind. Um aus diesen Werte abzuleiten, welche den Schmelzwärmen der Mischkristalle proportional sind, wurden dieselben durch die Gewichtsmengen der Schmelzen (Tabelle 1) dividiert und in der Kolonne „Mittelwert pro g“" (Tabelle 3) verzeichnet. Man sieht, dafs die den Schmelzwärmen proportionalen Werte angenähert linear mit wachsendem Siliciumgehalt ansteigen.

Bei Schmelzen mit höherem Siliciumgehalt tritt in den $\mathrm{Ab}$ kühlungs- und Erhitzungskurven eine zweimalige Verzögerung der Abkühlungs- resp. Erhitzungsgeschwindigkeit auf. Die erhaltenen Daten sind in den Tabellen 4 und 5 wiedergegeben. Tabelle 4 beschreibt die primäre Kristallisation im Konzentrationsintervall von 34.7 bis zu 98 Atomproz. Silicium, Tabelle 5 die sekundäre. Dem-

Tabelle 4 .

Primäre Kristallausscheidung.

\begin{tabular}{|c|c|c|c|c|c|c|}
\hline \multicolumn{2}{|c|}{ Gehalt an $\mathrm{Si}$} & \multicolumn{2}{|c|}{ Geschwindigkeit der } & \multicolumn{3}{|c|}{ Temperaturen } \\
\hline $\begin{array}{l}\text { in } \\
\text { Atom- } \\
\text { prozenten }\end{array}$ & $\begin{array}{c}\text { in } \\
\text { Gewichts- } \\
\text { prozenten }\end{array}$ & $\begin{array}{c}\text { Abküh- } \\
\text { lung } \\
a\end{array}$ & $\begin{array}{c}\text { Er- } \\
\text { litzung } \\
e\end{array}$ & $\begin{array}{l}\text { d. Beginns d. } \\
\text { Ausseheidg. } \\
\text { bei der Ab- } \\
\text { kühlung } t_{a}\end{array}$ & $\begin{array}{l}\text { des Endes } \\
\text { d. Schmelzg. } \\
\text { bei der Er- } \\
\text { hitzung } t_{e}\end{array}$ & $t_{0}$ \\
\hline 34.7 & 21.3 & 1.65 & 1.65 & - & -- & - \\
\hline 38.6 & 24.4 & 1.80 & 1.50 & 1365 & 1370 & 1368 \\
\hline 42.4 & 27.3 & 1.75 & 2.50 & $>1350$ & 1420 & 1400 \\
\hline 47.5 & 31.6 & 2.00 & 0.70 & 1425 & 1445 & 1435 \\
\hline $\begin{array}{l}50.0 \\
\text { Verbindu }\end{array}$ & $\begin{array}{l}33.7 \\
\text { ung }\end{array}$ & 1.75 & 1.40 & 1430 & 1455 & 1443 \\
\hline 54.9 & 38.3 & 2.00 & 1.20 & 1435 & 1450 & 1442 \\
\hline 58.1 & 41.5 & 2.50 & & 1405 & & 1415 \\
\hline 68.7 & 52.8 & 1.20 & 1.50 & 1340 & 1365 & 1352 \\
\hline 78.1 & 64.6 & 2.00 & 2.40 & 1275 & 1285 & 1279 \\
\hline 84.2 & 73.2 & 1.65 & 1.05 & 1350 & 1370 & 1360 \\
\hline 88.6 & 79.8 & 1.75 & 1.70 & 1375 & 1400 & 1388 \\
\hline 98 & 98 & 1.80 & 1.75 & 1420 & 1430 & 1425 \\
\hline
\end{tabular}




\begin{tabular}{|c|c|c|c|c|}
\hline \multirow{2}{*}{ 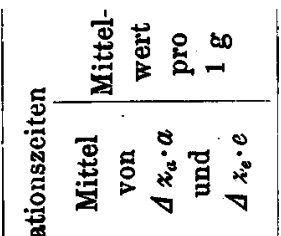 } & 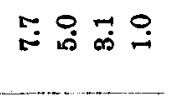 & & 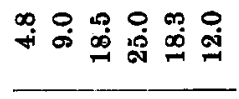 & \\
\hline & 栗 & & 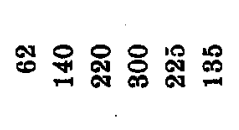 & \\
\hline 䎏 & 요욱 워우 & & 누용응영용 & \\
\hline$\dot{\nabla}$ & 요융ㅇ & & 용요욤욤 & \\
\hline 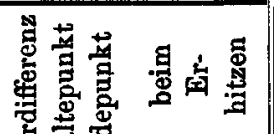 & 유옹오으 & & 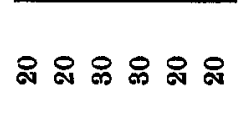 & \\
\hline 要 & 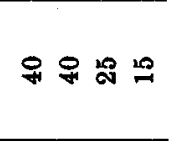 & & 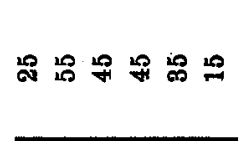 & \\
\hline 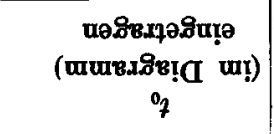 & 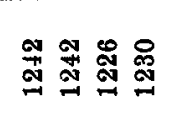 & & 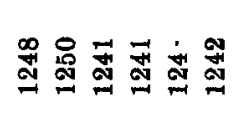 & \\
\hline 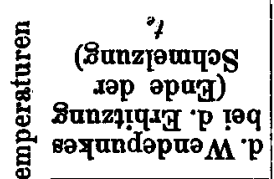 & 造总怘 & & 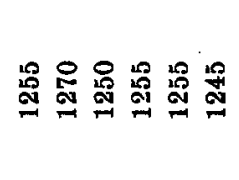 & \\
\hline 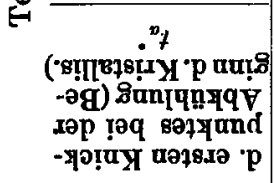 & 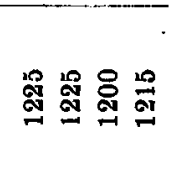 & & 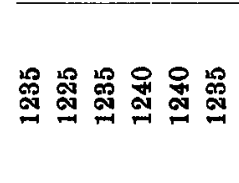 & \\
\hline 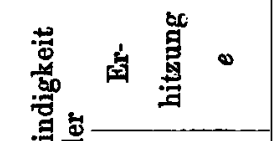 & 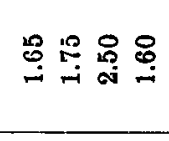 & 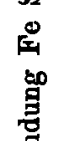 & 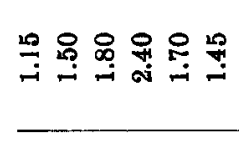 & $\widehat{\mathrm{g}}$ \\
\hline 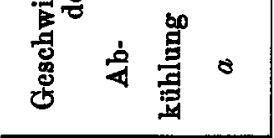 & 过 8 & 㕠 & 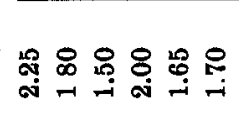 & 总 \\
\hline 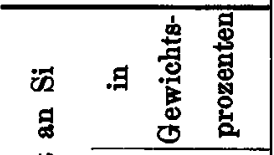 & 禹 & 俤 & 禹 & $\stackrel{\infty}{\infty}$ \\
\hline 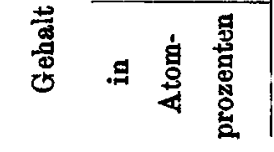 & 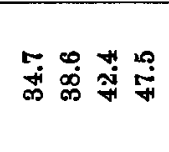 & 官 & 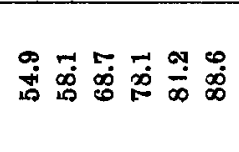 & $\stackrel{\infty}{\infty}$ \\
\hline
\end{tabular}


gemäls enthält Tabelle 4 nur die Abkühlungs- und Erhitzungsgeschwindigkeit, $a$ und $e$, und die Temperaturen der Knicke, $t_{a}$, $t_{e}$, und $t_{o}$.

Die Kristallisationszeiten in der Kolonne „Mittelwert pro g“ (Tabelle 5) sind den Mengen, die bei den eutektischen Temperaturen $1235^{\circ}$ und $1245^{\circ}$ kristallisieren, angenähert proportional. Es würde sich empfehlen, in Fällen, wo die spezifischen Volumina der beiden Komponenten sehr verschieden sind, die eingewogenen Mengen so zu bemessen, dafs man immer gleiche Volumina der verschiedenen Schmelzen in Tiegeln gleicher Form bei gleicher Länge des Thermoelementes hat.

\section{Das Zustandsdiagramm.}

In Figur 1 sind die Temperaturen des Beginnes und des Endes der Kristallisation aus den Tabellen 3,4 und 5 eingetragen. Mit steigendem Siliciumgehalt wächst bis zu etwa 15 Atomproz. Si das Kristallisationsintervall und nimmt dann bis etwa 30 Atomproz. ab. Der Beginn der Kristallisation ist durch die Kurve $a d b$ und das Ende durch die Kurve $a c b$ beschrieben. Die Abkühlungskurve einer Schmelze mit 33 Atomproz. Silicium hat dieselbe Form wie die des reinen Eisens. Es verhält sich jene Schmelze also in dieser Beziehung wie eine chemische Verbindung. Aufserdem entspricht ihre Zusammensetzung dem Gesetz der multiplen Proportionen, nämlich der Formel $\mathrm{Fe}_{2} \mathrm{Si}$. Man kann den Kristall von der $\mathrm{Zu}$ sammensetzung $\mathrm{Fe}_{2} \mathrm{Si}$ als einen gesättigten Mišchkristall oder als eine chemische Verbindung ansprechen. Zugunsten der letzteren Auffassung spricht vor allen Dingen die Tatsache, dafs alle Mischkristalle dieser Reihe, von $30-0 \% \mathrm{Si}$, beim Behandeln mit Säuren nach den Angaben von Hahn, Gin, OSMond, Morssan, Lebeau, Carnot und Goutal (l. c.) Rückstände derselben Zusammensetzung, die der Formel $\mathrm{Fe}_{2} \mathrm{Si}$ entsprechen, hinterlassen. Wären diese Mischkristalle als Mischungen von Eisen und Silicium, oder von $\mathrm{Fe}$ mit einer siliciumreicheren Verbindung aufzufassen, so wäre zu erwarten, dâs die beim Behandeln mit Säuren hinterbleibenden Rückstände entweder aus Silicium oder einer siliciumreicheren Verbindung bestehen. Der aus den Abkühlungskurven gezogene Schlufs, dafs die Schmelzen von $0-33 \%$ Silicium als eine kontinuierliche Reihe von Mischkristallen erstarren, wird durch die Struktur der Reguli bestätigt. Beim Zerschlagen zerspringen dieselben zu Würfeln von $1-4 \mathrm{~mm}$ Kantenlänge. Die Schlifffächen dieser Reguli werden von 
verdünnter Salzsäure gleichmälsig angegriffen und bestehen offenbar aus einem einzigen Strukturelement. Trotz verhältnismälsig schneller Abkühlung treten hier nicht wie bei den Manganstahlen zwei Strukturelemente auf, sondern die Reguli bestehen aus einer einzigen Kristall-

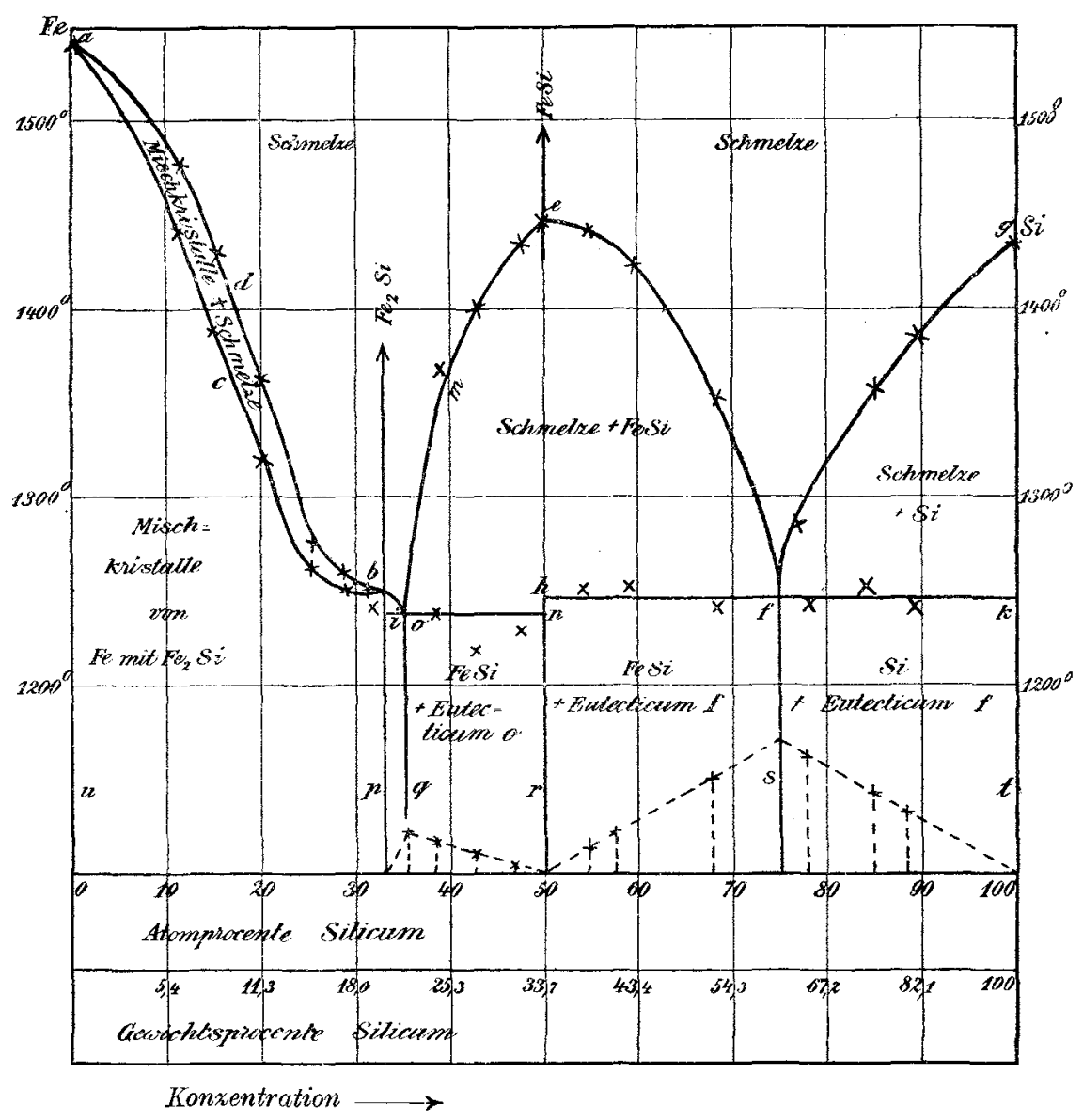

Fig. 1. Zustandsdiagramm.

art. Zwischen 20 und $33 \%$ Si finden sich in den Reguli zahlreiche kleine Hohlräume, welche die einzelnen Kristallpolyeder voneinander trennen, wie in Figur 1 Tafel II deutlich zu sehen ist.

Die Kurve mef gibt die Temperaturen der Ausscheidung einer neuen Kristallart an. Bei $1443^{\circ}$ und zwischen 47.5 und 54.9 Atomproz. hat dieselbe ein Maximum. Durch Interpolation findet man dasselbe zwischen 49.3 und 52.6 Atomproz. Si. Die 
Formel FeSi verlangt 50.0 Atomproz. Benutzt man die Zeitdauern der Kristallisation, bezogen auf gleiche Gewichtsmengen Substanz, zur Bestimmung der Zusammensetzung der Verbindung (in Fig. 1 sind die Ordinaten der gestrichelten Linien proportional jenen Werten) und extrapoliert man aus je zwei Werten der Kristallisationsdauer, bei der diese Zeitdauer null wird, so findet man folgende Werte für die Zusammensetzung der Verbindung:

Aus der Kristallisationsdauer

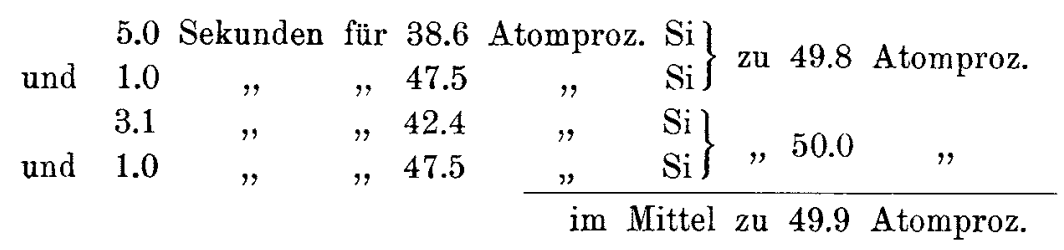

\begin{tabular}{|c|c|c|c|c|c|c|c|c|}
\hline & 4.8 & Sekunden & für & 54.9 & Atomproz. & $\mathrm{Si}$ & 711 & Atomproz. \\
\hline und & 9.0 & $\eta$ & $"$ & 58.1 & $"$ & $\mathrm{Si}\}$ & 2401.1 & 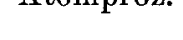 \\
\hline & 4.8 & " & " & 54.9 & $"$ & $\mathrm{Si}\}$ & 50.1 & \\
\hline & 18.5 & $"$ & , & 68.7 & , & $\mathrm{Si} J$ & " & $"$ \\
\hline
\end{tabular}

Aus diesen beiden Mittelwerten würde also die Zusammensetzung der Verbindung zu 50.2 Atomproz. Si folgen. Man darf wohl auf Grund dieser Bestimmungen annehmen, dafs die Zusammensetzung der zweiten Verbindung der Formel FeSi mit 50.0 Atomproz. gleich 33.7 Gewichtsproz. Silicium entspricht. Diese Annahme wird durch die Gestalt der Abkühlungskurve einer Schmelze mit 50.0 Atomproz. Si bestätigt. Auf dieser Kurve findet sich bei $1430^{\circ}$ ein Haltepunkt, während jede Andeutung eines solchen bei den Temperaturen der eutektischen Horizontalen in und $h f k$ fehlt. Fügt man zu dieser Schmelze entweder $\mathrm{Si}$ oder $\mathrm{Fe}$, so wird, wie den Abkühlungskurven zu entnehmen ist, die Temperatur des Beginnes der Kristallisation erniedrigt und die Menge, welche sich bei dieser Temperatur ausscheidet, verkleinert. Dafür wachsen die Mengen, welche bei den eutektischen Temperaturen $i n$ und $h f k$ kristallisieren. Durch Extrapolation findet man, dafs dieselben etwa bei 76 Atomproz. Si ein Maximum erreichen und von da an wieder zum reinen Silicium hin abnehmen.

Entsprechend dem Verlauf der Kurve mef und der Existenz der Verbindung $\mathrm{Fe}_{2} \mathrm{Si}$ war zu erwarten, dals die Reguli von 33.3 
bis 50.0 Atomproz. Silicium aus primär ausgeschiedenen Kristallen der Verbindung $\mathrm{FeSi}$, umgeben von den Kristallen der Verbindung $\mathrm{Fe}_{2} \mathrm{Si}$, bestehen würden. Es war also zu erwarten, dafs die Kristalle der Verbindung FeSi von einem homogenen Strukturelement umgeben wären. Nun ergab sich aber, wie in Fig. 3 und 4 (Tafel II) dentlich zu sehen ist, dafs das Strukturelement, welches die primär ausgeschiedenen Kristalle der Verbindung FeSi umgibt, nach dem Anätzen mit Salzsäure deutlich eine eutektische Struktur zeigt. Fig. 2 (Tafel II) stellt die Struktur dieses Eutektikums in einem Regulus mit 34.7 Atomproz. Si in 200 facher Vergröfserung dar.

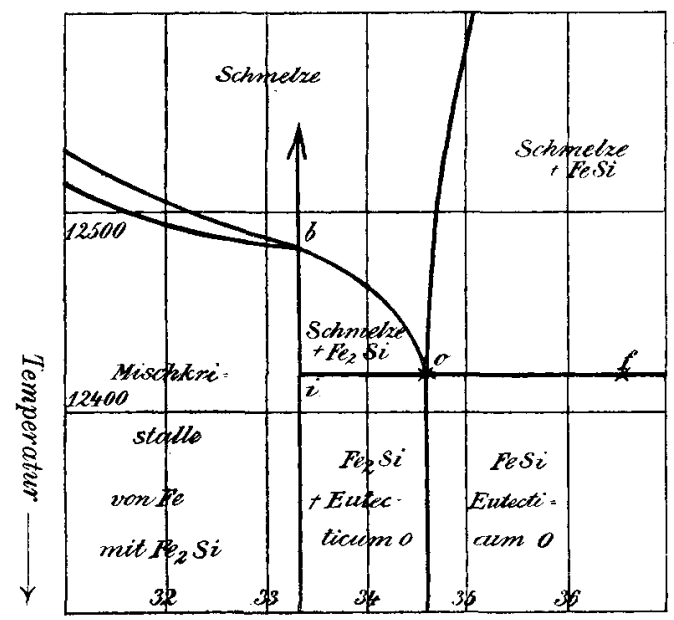

Konzentration in Atomproz. Si

Fig. 2.

Dieser Regulus besteht ausschliefslich aus dem Eutektikum, dessen Menge mit steigendem Si-Gehalt abnimmt und bei 50 Atomproz. Silicium verschwindet. Diese Tatsachen führen zur Annahme der Existenz eines sehr kurzen Schmelzkurvenastes $b o$. Der Punkt $o$ ist nahe bei 34.7 Atomproz. Si anzunehmen, weil der Regulus dieser Zusammensetzung nur aus dem Eutektikum besteht, während im Regulus mit 38.6 Atomproz. Si (Fig. 3 auf Tafel II) schon eine erhebliche Menge der Verbindung FeSi vorhanden ist. In der Spezialfigur 2 erkennt man deutlicher den Kurvenast $b o$ und die unterhalb desselben anzunehmende eutektische Gerade $i o$. Da sich die Kurve $b o$ nur über ein Konzentrationsintervall von etwa $2 \%$ und über ein Temperaturgebiet von höchstens $8^{\circ}$ erstrecken kann, so ist es 
natürlich nicht möglich, die Existenz dieses Kurvenastes auf Grundlage von Schmelzpunktsbestimmungen zu erweisen.

Die Kurve $g f$ Fig. 1 ist die Löslichkeitskurve des kristallisierten Siliciums, welche den Kurvenast $e f$ im eutektischen Punkte $f$ bei 76 Atomproz. Si trifft. Bei der Abkühlung scheidet sich auf dieser Kurve das Silicium primär aus, und wird dann ron einem Eutektikum, welches aus $\mathrm{Si}+\mathrm{SiFe}$ besteht, umgeben, wie in Fig. 6 Tafel 1, in der die langen Siliciumkristalle, umgeben von Eutektikum, zu sehen sind. Beim Schleifen dieser Reguli bröckelt das Eutektikum aufserordentlich leicht aus, wodurch die vielen schwarzen Löcher in Fig. 6 entstanden sind.

Fig. 5 Tafel 1 gibt die Struktur eines Regulus, der etwa $49 \% \mathrm{Si}$ enthält, nach dem Ätzen mit Natronlauge. Die Verbindung FeSi ist angegriffen worden, während das Eutektikum, aufser dieser die Verbindung $\mathrm{Fe}_{2} \mathrm{Si}$ enthaltend, viel weniger angegriffen ist.

Im folgenden sind die Zustandsfelder der verschiedenen Eisensiliciumlegierungen zusammengestellt. Die polymorphen Umwandlungen des Eisens und seiner Mischkristalle sind hierbei nicht berücksichtigt worden. In diesem Punkte ist noch eine ergänzende Untersuchung auszuführen.

Die Zustandsfelder oberhalb $1100^{\circ}$ sind:

I. Das Zustandsfeld der Schmelze oberhalb des Kurvenzuges adbo efg.

II. Die Zustandsfelder, in denen eine Kristallart mit der Schmelze im Gleichgewicht ist:

1. Mischkristalle von $\mathrm{Fe}$ und $\mathrm{Fe}_{2} \mathrm{Si}$

innerhalb des Feldes $a d b c a$ von 0-33.3 Atomproz. Si

2. die Verbindung $\mathrm{Fe}_{2} \mathrm{Si}$

innerhalb des Feldes $b$ o $i$ von 33.3-35 " "

3. die Verbindung $\mathrm{FeSi}$

innerhalb des Feldes oefhno von $35-76$

4. reines $\mathrm{Si}$ innerhalb des Feldes $g f k$ von $76-100$

III. Die Zustandsfelder mit einer Kristallart:

Mischkristalle von $\mathrm{Fe}$ mit $\mathrm{Fe}_{2} \mathrm{Si}$ innerhalb des Feldes $u a b p$ von 0-33.3 Atomproz. Si.

IV. Die Zustandsfelder mit zwei Kristallarten:

1. $\mathrm{Fe}_{2} \mathrm{Si}$ mit Eutektikum $o$

innerhalb des Feldes pioq von 33.3-35 Atomproz. Si 
2. FeSi mit Eutektikum 0

innerhalb des Feldes 9 on $r$ von $35-50$ Atomproz. Si

3. FeSi mit Eutektikum $f$

innerhalb des Feldes $r h f s$ von 50-76 " "

4. Si mit Eutektikum $f$

innerhalb des Feldes $s f k t$ von $76-100 \quad " \quad$,

Das Verhalten der hier vorkommenden Kristallarten gegen Mineralsäuren und Alkalien ist folgendes: Silicium wird von heifser Kalilauge schnell angegriffen, die Verbindung FeSi viel langsamer, kaum merklich die Verbindung $\mathrm{Fe}_{2} \mathrm{Si}$ und $\mathrm{Fe}$. Umgekehrt ist das Verhalten der Salzsäure, welche Eisen schnell, $\mathrm{Fe}_{2} \mathrm{Si}$ erheblich langsamer, FeSi und Si nicht merklich angreift. Das Verhalten von $\mathrm{Si}$ und der Verbindung FeSi zu Natronlauge kann man zur Isolierung der letzteren aus den Reguli mit mehr als 50 Atomproz. Si benutzen. Ein Regulus mit 80 Atomproz. Si wurde mit kalter Natronlauge behandelt, wobei derselbe in zwei Tagen zu einem Pulver zerfiel, in dem 50.1 Atomproz. Fe und 50.7 Atomproz. Si gefunden wurden.

Betreffs der Härte der verschiedenen Kristallkonglomerate ergab sich, dafs dieselbe mit wachsendem Eisengehalt abnimmt. Am härtesten ist das Silicium (etwas härter als Quarz), nur wenig weicher die Verbindung $\mathrm{FeSi}$. Während die Verbindung $\mathrm{Fe}_{2} \mathrm{Si}$ etwa Apatithärte hat, nimmt die Härte der Mischkristalle mit steigendem Eisengehalt stark bis zur Flufsspathärte ab.

In Tabelle 6 sind die auf gleiche Abkthlungs- und Erhitzungsgeschwindigkeit reduzierten Zeiten der Kristallisation für die bei konstanter Temperatur kristallisierenden Schmelzen zusammengestellt. Die auf gleiche Gewichtsmengen bezogenen Mittelwerte sind den Schmelzwärmen pro Masseneinheit angenähert proportional. Man

Tabelle 6.

\begin{tabular}{c|c|c|c|c|c|c}
\hline & $\begin{array}{c}\text { Schmelz- } \\
\text { punkte }\end{array}$ & $\Delta \pi_{a} \cdot a$ & $\Delta x_{e} \cdot e$ & Mittel & $\begin{array}{c}\text { Verhältnis- } \\
\text { zahlen } \\
\text { für Fe }=1\end{array}$ & $\begin{array}{c}\text { Auf gleiche } \\
\text { Gewichts- } \\
\text { menge } \\
\text { umgerechnet }\end{array}$ \\
\hline Fe & 1540 & 115 & 110 & 112 & 1 & 1 \\
FesSi & 1251 & 120 & 100 & 110 & 1.04 & 1.15 \\
Eutektikam o & 1235 & 130 & 130 & 130 & 1.16 & 1.25 \\
FeSi & 1443 & 255 & 245 & 250 & 2.2 & 2.9 \\
Eutektikum $f$ & 1245 & graphisch extrapol. & 320 & 2.8 & 4.5 \\
Si & 1425 & 380 & - & 380 & 3.3 & 6.6
\end{tabular}

Z. anorg. Chem. Bd. 47 . 
ersieht, dafs die Schmelzwärme vom Silicium bis zum Eisen sehr erheblich abnimmt.

Bei den Reguli von 0 bis 47.5 Atomproz. Silicium war auf eine empfindliche Magnetnadel eine starke Wirkung, deren Stärke mit dem Siliciumgehalt deutlich abnahm, zu beobachten, während Reguli mit mehr als 50 Atomproz. Silicium auch auf eine empfindliche Magnetnadel keine Wirkung ausübten. Dieses Verhalten stimmt mit den Angaben von Jouve ${ }^{1}$, welcher die Kräfte, mit denen gleiche Massen dieser Legierungen von einem starken Elektromagneten angezogen wurden, bestimmte, überein. Jouve fand, dals die magnetische Permeabilität mit steigendem Siliciumgehalt abnimmt und zwar nicht kontinuierlich. Bei 33.3 und bei 50.0 Atomproz. Silicium finden sich Diskontinuitäten.

Bekanntlich vermindert sich die magnetische Permeabilität bei der Umwandlung des $\alpha$-Eisens in $\beta$-Eisen (bei $760^{\circ}$ ) ganz aufserordentlich. Ein Rest der Permeabilität bleibt aber auch bei höheren Temperaturen zurück. Läfst man ein Stück Eisen in der. Nähe einer nicht zu empfindlichen Magnetnadel abkühlen, so findet man, dafs bei $760^{\circ}$ die magnetische Permeabilität plötzlich auftritt, dals also die Magnetnadel auf das Eisen plötzlich zu wirken beginnt. Nun ergab sich bei der Feststellung der Temperatur des Verlustes der Permeabilität der Siliciumeisenlegierungen, dafs derselbe sehr angenähert, wie aus folgender Zusammenstellung ersichtlich ist, bei derselben Temperatur eintrat. Es wäre daraus zu schliefsen, dafs die Gegenwart von Si die Umwandlungstemperatur des $\beta$-Eisens in $\alpha$-Eisen nicht merklich beeinflufst.

Tabelle 7.

\begin{tabular}{c|c}
\hline \hline Atomprozent Si & Umwandlungstemperatur \\
\hline 0.06 & $760 \pm 5$ \\
10.4 & $750 \pm 10$ \\
20.0 & $745 \pm 10$ \\
29.1 & $735 \pm 10$ \\
38.6 & $770 \pm 10$ \\
47.5 & $720 \pm 20$
\end{tabular}

1 Compt. rend. 134 (1902), 1577. 
Die thermischen Erscheinungen bei der Kristallisation der Eisensiliciumschmelzen stimmen also sowohl mit der Struktur der Legierungen als auch mit den physikalischen Eigenschaften befriedigend überein. Dieselben werden bedingt durch die Bildung zweier Verbindungen $\mathrm{FeSi}$ und $\mathrm{Fe}_{2} \mathrm{Si}$, von denen die letztere in dem Zustandsdiagramm der Eisensiliciumlegierungen eine ganz besondere, beispiellose Stellung als Endglied einer Reihe von Mischkristallen einnimmt.

Qöttingen, Institut für anorganische Chemie der Universität.

Bei der Redaktion eingegangen am 15. August 1905. 


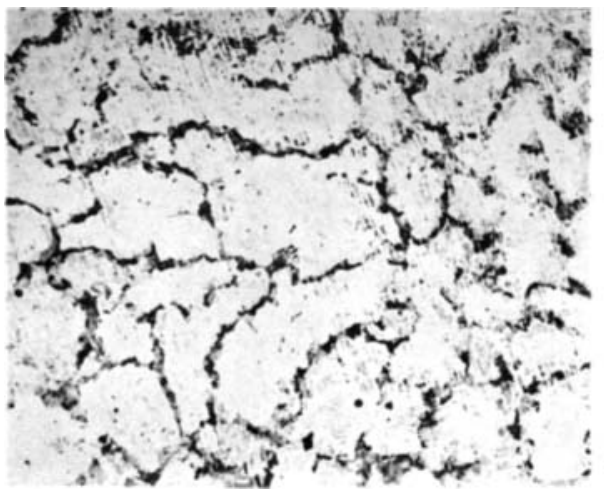

Fig. 1.

68,8 Atomproc. Fe. + 31,2 Atomproc. Si. 40 fache Vergrösserung.

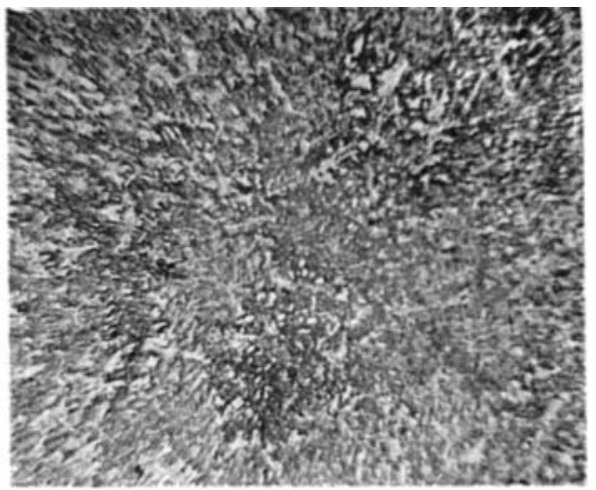

Fig. 2.

65,3 Atomproc. $\mathrm{Fe},+34,7$ Atomproc. $\mathrm{Si}$. 200 fache Vergrösserung.

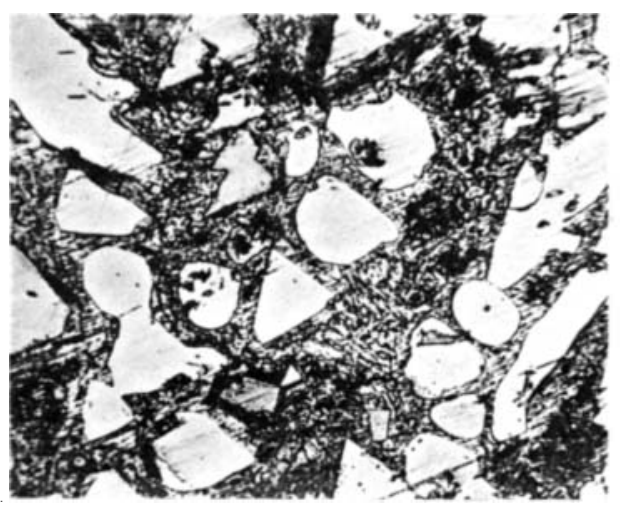

Fig. 3.

61,4 Atomproc. Fe. $+38,6$ Atomproc. Si. 100 fache Vergrösserung.

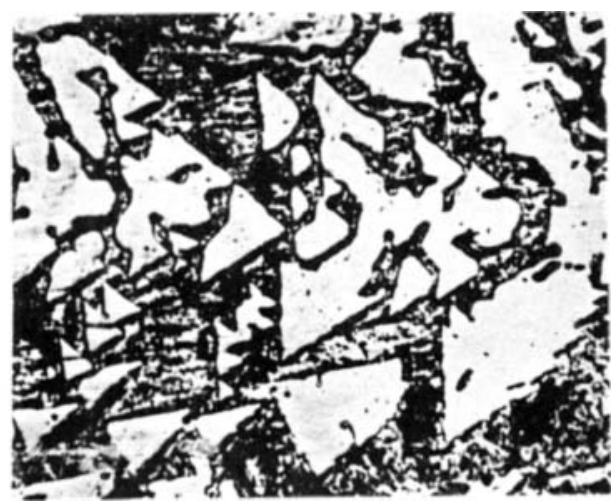

Fig. 4.

57,6 Atomproc. Fe. $+42,4$ Atomproc. Si. 80 fache Vergrösserung.

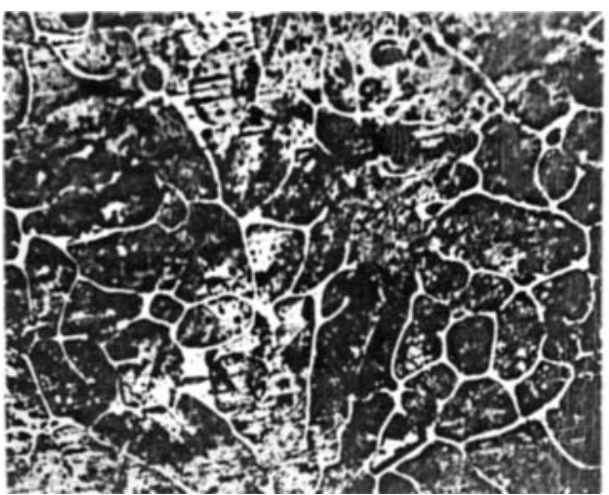

Fig. 5.

50 Atomproc. Fe. +50 Atomproc. Si. 50 fache Vergrösserung.

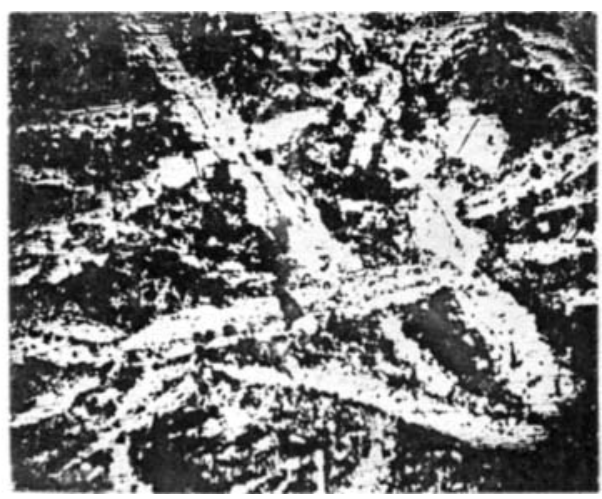

Fig. 6.

15,8 Atomproc. Fe. $+84,2$ Atomproc. $\mathrm{Si}$. 40 fache Vergrösserung. 